\title{
A PRODUCT INTEGRAL SOLUTION OF A STIELTJES-VOLTERRA INTEGRAL EQUATION
}

\author{
JAMES A. RENEKE
}

Abstract. This paper demonstrates that the theory of StieltjesVolterra integral equations may be subsumed in Mac Nerney's general integral equation theory by making suitable choices of linear spaces and sets of operators.

Introduction. Our object is to obtain solutions of nonlinear integral equations of the form $h(t)=f(t)+(L) \int_{c}^{t} d F[t, \quad] h$ using the theory of the product integral developed by Mac Nerney and Neuberger in [3], [4], and [5]. This approach specialized to the linear case may be contrasted with that of Hinton [1].

Suppose that $S$ is a compact number-interval with the usual order denoted by $\mathcal{O}$ and $\{X,\|\cdot\|\}$ is a complete normed linear space with zero element 0 . Let $M$ denote the class of all functions from $X$ to $X$ to which $\{0,0\}$ belongs and 1 the identity function in $M$. Let $F_{1}$ be the set of all functions $F$ from $S \times S$ into $M$ such that

(1) $F(t, t)=1$ for all $t$ in $S$,

(2) $F[, t] P$ is quasi-continuous for each $t$ in $S$ and $P$ in $X$, and

(3) there is a nondecreasing function $k$ on $S$, called a super function for $F$, such that

$$
\|\{F(t, u)-F(t, v)\} P-\{F(t, u)-F(t, v)\} Q\| \leqq|k(u)-k(v)| \cdot\|P-Q\|
$$

for all $t, u, v$ in $S$ and $P$ and $Q$ in $X$. Finally, let $\digamma_{2}$ denote the class of all functions from $S$ into $X$ of bounded variation and $\mathcal{F}_{3}$ the class of all functions from $S$ into $X$ which are quasi-continuous.

The existence of a solution. Suppose that $F$ is a member of $F_{1}$ with super function $k, f$ is a member of $\mathscr{F}_{3}$, and $c$ is a member of $S$. We want to establish the existence of a function $h$ in $\Im_{3}$ such that

$$
h(t)=f(t)+(L) \int_{c}^{t} d F[t,] h,
$$

for each $t$ in $S$, where the integral is the left integral whose definition is analogous to that of the right integral in [4]. For each $u$ in $S$ let $A_{u}$

Presented to the Society, November 22, 1969; received by the editors April 19,

A MS Subject Classifications. Primary 4513, 4530; Secondary 4750.

Key Words and Phrases. Product integral, Stieltjes-Volterra integral equations, left integral, right integral, order additive functions, order multiplicative functions. 
denote the function from $\mathcal{F}_{3}$ into the class of all functions from $S$ into $X$ defined by $A_{u}(g)(t)=(L) \int_{c}^{x} d F[t, \quad] g$.

THEOREM 1. $A_{u}$ is a function from $\mathcal{F}_{3}$ into $\mathfrak{F}_{3}$ which contains $\{0,0\}$, where 0 is used to denote the element of $\mathcal{F}_{3}$ whose only value is 0 .

We can note that, for each $g$ in $\mathcal{F}_{3}$ and $t$ in $S,(L) \int_{c}^{u} d F[t, \quad] g$ exists [4] and, furthermore,

$\left\|(L) \int_{c}^{u} d F[t] g,\right\| \leqq|k(u)-k(c)| \cdot m$,

where $m=1$.u.b. $[\|g(v)\|: v=c$ or $v=u$ or $v$ is between $c$ and $u]$.

Thus $A_{u}$ contains $\{0,0\}$.

Lemma. If $g$ is in $\digamma_{3}$ and $\epsilon$ is a positive number, then there is an $\mathcal{O}$-partition $r$ of $\{c, u\}$ such that

$$
\left\|(L) \int_{c}^{u} d F[t,] g-(L) \sum_{s} d F[t,] g\right\|<\epsilon,
$$

for each O-refinement $s$ of $r$ and $t$ in $S$.

Proof. By the remark preceding the lemma and the fact that each element of $\mathscr{F}_{3}$ is the uniform limit of a sequence with values in $\mathscr{F}_{2}$ [2], if we establish the lemma for $g$ in $F_{2}$ we are through. Assume that $c<u$ and $g$ is in $\digamma_{2}$. Since $(L) \int_{c}^{u} k d h$ exists [3], where $h$ is the function from $[c, u]$ to the nonnegative numbers defined by $h(v)=\int_{c}^{v}|d g|$, for all $v$ in $[c, u]$, there is an $\mathcal{O}$-partition $\left\{r_{p}\right\}_{0}^{n}$ of $\{c, u\}$ such that if $\left\{s_{p}\right\}_{0}^{m}$ is an $\mathcal{O}$-refinement of $r, \alpha$ is a nondecreasing integer valued sequence, and $r=s[\alpha]$ then

$$
\sum_{p=1}^{n} \sum_{q=\alpha(p-1)+1}^{\alpha(p)}\left(k\left(s_{q}\right)-k\left(s_{q-1}\right)\right) \int_{r(p-1)}^{8(q-1)}|d g|<\epsilon .
$$

Hence

$$
\begin{aligned}
& \| \sum_{p=1}^{n} \sum_{q=\alpha(p-1)+1}^{\alpha(p)}\left\{\left[F\left(t, s_{q}\right)-F\left(t, s_{q-1}\right)\right] g\left(r_{p-1}\right)\right. \\
&\left.-\left[F\left(t, s_{q}\right)-F\left(t, s_{q-1}\right)\right] g\left(s_{q-1}\right)\right\} \|<\epsilon
\end{aligned}
$$

for each $t$ in $S$. Thus we have the lemma.

Proof of THE THEOREM. If $\epsilon$ is a positive number, $\left\{s_{p}\right\}_{0}^{n}$ is an 0 -partition of $\{c, u\}$ as in the lemma, and each of $v$ and $w$ is in $S$ then 


$$
\begin{aligned}
\| A_{u}(g)(v)- & A_{u}(g)(w) \| \\
\leqq & +\sum_{p=1}^{n}\left\|\left\{F\left(v, s_{p}\right)-F\left(w, s_{p}\right)\right\} g\left(s_{p-1}\right)\right\| \\
& \quad+\sum_{p=1}^{n}\left\|\left\{F\left(v, s_{p-1}\right)-F\left(w, s_{p-1}\right)\right\} g\left(s_{p-1}\right)\right\| .
\end{aligned}
$$

Hence we have the theorem.

We will use the notation of [4]. Take $\mathcal{F}_{3}$ as the abelian group $G$ and norm it with the supremum norm $\|\cdot\|$. Let $H$ denote the class of all functions from $G$ into $G$ to which $\{0,0\}$ belongs and let 1 denote the identity function in $H$. We will depend on the context to distinguish between the zeros of $X, M$, and $H$ and the identities of $M$ and $H$. Specializing Mac Nerney's definitions to our situation, we have $\theta a^{+}$ is the class of $\theta$-additive functions from $S \times S$ into the nonnegative numbers; $\mathcal{O N}^{+}$is the class of $\theta$-multiplicative functions from $S \times S$ into the numbers no smaller than $1 ; O Q$ is the class of functions from $S \times S$ into $H$ to which $V$ belongs only in case

(1) $V$ is $O$-additive and

(2) there is a member $\alpha$ of $O Q^{+}$such that if $\{u, v\}$ is in $S \times S$ and $\{f, g\}$ is in $G \times G$ then $\|V(u, v) f-V(u, v) g\| \leqq \alpha(u, v)\|f-g\|$; and $\mathcal{O N} \boldsymbol{F}$ is the class of functions from $S \times S$ into $H$ to which $W$ belongs only in case

(1) $W$ is $\theta$-multiplicative and

(2) there is a member $\mu$ of $\mathcal{O N T ^ { + }}$ such that if $\{u, v\}$ is in $S \times S$ and $\{f, g\}$ is in $G \times G$ then $\|[W(u, v)-1] f-[W(u, v)-1] g\| \leqq(\mu(u, v)-1)$ $\|f-g\|$.

Theorem 2. The function $V$ from $S \times S$ into $H$ defined by

$$
[V(u, v) f](t)=A_{u}(f)(t)-A_{v}(f)(t)=(L) \int_{v}^{u} d F[t, \text { lf, for all } t \text { in } S,
$$

is an element of $\mathcal{O}$.

Proof. For each $u, v$, and $t$ in $S, g$ and $h$ in $G$, and positive number $\epsilon$,

$$
\begin{aligned}
\|[V(u, v) g](t)-[V(u, v) h](t)\| & =\left\|(L) \int_{v}^{u} d F[t,] g-(L) \int_{v}^{u} d F[t,] h\right\| \\
& \leqq \epsilon+|k(v)-k(u)| \cdot\|g-h\| .
\end{aligned}
$$

Thus $V$ is in 0 a.

Let $W$ be the function from $S \times S$ into $H$ defined by $W(t, u) g$ $={ }_{i} \Pi^{u}[1+V] g$, for each $\{t, u\}$ in $S \times S$ and $g$ in $G$ [4, Theorem 1.1]. 
Then $W$ is in OTK and $W(t, u) g=g+(R) \int_{t}^{u} V W[, u] g$, for each $\{t, u\}$ in $S \times S$, where the integral is the right integral [4, Corollary 2.1]. We will take as our candidate for a solution of $\left(^{*}\right)$ the function $h$ defined by $h(u)=[W(u, c) f](u)$, for each $u$ in $S$.

THEOREM 3. $h$ is in $\mathfrak{F}_{3}$.

Proof. Note that the function $g$ from $S$ into $G$ defined by $g(u)$ $=W(u, c) f$ has bounded variation on $S$ and hence is quasicontinuous. Suppose that $\epsilon$ is a positive number, $t$ is in $S$, and $t$ is not a right-hand endpoint of $S$. There is a positive number $d_{1}$ such that if each of $u$ and $v$ is in $\left(t, t+d_{1}\right)$ then $\|W(u, c) f-W(v, c) f\|<\epsilon / 3$. Let $w$ be a point of $\left(t, t+d_{1}\right)$. There is a positive number $d_{2}$, smaller than $d_{1}$, such that if each of $u$ and $v$ is in $\left(t, t+d_{2}\right)$ then $\left.\| W(w, c) f\right](u)-[W(w, c) f](v) \|$ $<\epsilon / 3$. Hence if $u$ and $v$ are in $\left(t, t+d_{2}\right)$ then

$$
\begin{aligned}
\|h(u)-h(v)\| \leqq & \|[W(u, c) f](u)-[W(w, c) f](u)\| \\
& +\|[W(w, c) f](u)-[W(w, c) f](v)\| \\
& +\|[W(w, c) f](v)-[W(v, c) f](v)\|<\epsilon .
\end{aligned}
$$

Similarly, $h$ has a left-hand limit at each point of $S$ which is not a left-hand endpoint of $S$.

Theorem 4. $h(u)=f(u)+(L) \int_{c}^{u} d F[u, \quad] h$, for each $u$ in $S$.

Proof. We will establish the theorem by showing that $\left[(R) \int_{u}^{c} V W[, c] f\right](u)=(L) \int_{c}^{u} d F[u, \quad] h$, for each $u$ in $S$. Let $\alpha$ be a member of $\theta Q^{+}$such that $\|W(v, c) f-W(w, c) f\| \leqq \alpha(v, w)$ for each $\{v, w\}$ in $S \times S$. Suppose that $\epsilon$ is a positive number. There is an O-partition $\left\{r_{p}\right\}_{0}^{n}$ of $\{u, c\}$ such that

$$
\left\|\left[(R) \int_{u}^{c} V W[, c] f\right](u)+\sum_{p=1}^{m}(R) \int_{s(p-1)}^{s(p)} d F[u,] W\left(s_{p}, c\right) f\right\|<\epsilon / 8
$$

and $\left\|(L) \int_{c}^{u} d F[u, \quad] h+(R) \sum_{s} d F[u, \quad] h\right\|<\epsilon / 8$, for each $\theta$-refinement $\left\{s_{p}\right\}_{0}^{m}$ of $r$. There is an $\mathcal{O}$-refinement $\left\{s_{p}\right\}_{0}^{m}$ of $r$ and an increasing integer valued sequence $\beta$ such that $r=s[\beta]$,

$$
\begin{aligned}
\|(R) \int_{r(p-1)}^{r(p)} d F[u,] W\left[r_{p}, c\right] f \\
\quad-\sum_{q=\beta(p-1)+1}^{\beta(p)}\left\{F\left(u, s_{q}\right)-F\left(u, s_{q-1}\right)\right\}\left[W\left(r_{p}, c\right) f\right]\left(s_{q}\right) \|<\epsilon / 4 n,
\end{aligned}
$$

for $p=1,2, \cdots, n$, 
1970)

$$
\sum_{p=1}^{n} \sum_{q=\beta(p-1)+1}^{\beta(p)}\left|k\left(s_{q}\right)-k\left(s_{q-1}\right)\right| \alpha\left(r_{p}, s_{q}\right)<\epsilon / 4
$$

and

$$
\left\|(R) \sum_{r} d F[u,] h-(R) \sum_{\cdot} d F[u,] h\right\|<\epsilon / 4
$$

Therefore

$$
\begin{aligned}
& \left\|\left[(R) \int_{u}^{c} V W[, c] f\right](u)-(L) \int_{c}^{u} d F[u,] h\right\| \\
& <\epsilon / 4+\left\|\sum_{p=1}^{n}(R) \int_{r(p-1)}^{r(p)} d F[u,] W\left(r_{p}, c\right) f-(R) \sum_{r} d F[u,] h\right\| \\
& <\epsilon / 2+\| \sum_{p=1}^{n} \sum_{q=\beta(p-1)+1}^{\beta(p)}\left\{F\left(u, s_{q}\right)-F\left(u, s_{q-1}\right)\right\}\left[W\left(r_{p}, c\right) f\right]\left(s_{q}\right) \\
& \quad-(R) \sum_{r} d F[u,] h \| \\
& \quad-\left[W\left(s_{q}, c\right) f\right]\left(s_{q}\right)\|+\|(R) \sum_{\bullet} d F[u,] h-(R) \sum_{r} d F[u,] h \| \\
& \quad<3 \epsilon / 4+\sum_{p=1}^{n} \sum_{q=\beta(p-1)+1}^{\beta(p)}\left|k\left(s_{q}\right)-k\left(s_{q-1}\right)\right| \alpha\left(r_{p}, s_{q}\right)<\epsilon .
\end{aligned}
$$

Thus $\left[(R) \int_{u}^{c} V W[, c] f\right](u)=(L) \int_{c}^{u} d F[u, \quad] h$. Hence we have $h(u)$ $=f(u)+(L) \int_{c}^{u} d F[u, \quad] h$, for each $u$ in $S$.

Since $F$ is in $\mathcal{F}_{1}$ and has property (3), the proof of the uniqueness of the solution is the same as for the linear case [1, Theorem 3.1].

AN EXAMPLE. Consider the nonlinear delay equation $h(t)=f(t)$ $+\int_{a}^{t} g(I, h(I-\tau)) d I$ on the interval $S=[a, b]$. We can rewrite the equation as a Stieltjes-Volterra equation as follows:

$$
h(t)=f(t)+\int_{a \rightarrow \tau}^{t \rightarrow r} g(I+\tau, h) d I=f(t)+\int_{a \rightarrow \tau}^{t} d F_{1}[t,] h,
$$

where $F_{1}(t, u) x=\int_{a-\tau}^{0} g(I+\tau, x) d I$ and $v=\frac{1}{2}(t-\tau+u-|t-\tau-u|)$ for each number $x$ and ordered pair $(t, u)$ in $[a, b] \times[a-\tau, b]$. If $g[, x]$ is quasi-continuous on $S$ for each number $x$ and there is a number $k$ such that $|g(t, x)-g(t, y)| \leqq k|x-y|$ for each $t$ in $S$ and numbers $x$ and $y$, then $F_{1}$ satisfies conditions (2) and (3) of the introduction. Let 


$$
\begin{aligned}
F_{2}(t, u) & =F_{1}(t, u)-F_{1}(t, t)+1 & & \text { if } a<t \leqq b, \\
& =0 & & \text { if } a-\tau \leqq t \leqq a,
\end{aligned}
$$

for each $(t, u)$ in $[a-\tau, b] \times[a-\tau, b]$. Then $F_{2}$ satisfies conditions (1)-(3) and the equation becomes

$$
h(t)=\bar{f}(t)+\int_{a-\tau}^{t} d F_{2}[t,] h,
$$

where $\bar{f}$ is the initial function on $[a-\tau, a)$ and $f$ on $[a, b]$.

\section{REFERENCES}

1. D. B. Hinton, A Stieltjes-Volterra integral equation theory, Canad. J. Math. 18 (1966), 314-331. MR 32 \#6169.

2. R. E. Lane, The integral of a function with respect to a function, Proc. Amer. Math. Soc. 5 (1954), 59-66. MR 15, 514.

3. J. S. Mac Nerney, Integral equations and semigroups, Illinois J. Math. 7 (1963), 148-173. MR 26 \#1726.

4. - A nonlinear integral operation, Illinois J. Math. 8 (1964), 621-638. MR $29 \# 5082$.

5. J. W. Neuberger, Continuous products and nonlinear integral equations, Pacific J. Math. 8 (1958), 529-549. MR 21 \#1509.

\section{Clemson University}

